\title{
Critérios éticos e políticos para a triagem em tempos de crise: pensando a alocação de recursos escassos a partir da deficiência
}

\author{
Ethical and political criteria for triage in times of health crisis: considering the \\ allocation of scarce resources from disability
}

\author{
Luana Adriano Araújo* \\ Arthur Alves de Melo ${ }^{* *}$ \\ Ana Paula Barbosa-Fohrmann ${ }^{* * *}$
}

Resumo: A crise sanitária global provocada pela Covid-19 impôs um cenário crítico de escassez de recursos médicos. As pessoas com deficiência, nessas circunstâncias, são sujeitadas a maior vulnerabilidade do que ocorreria em circunstâncias normais. A luz dessa realidade, nos propomos a apresentar alguns critérios de alocação de recursos empregados durante a crise, destacando seu caráter nocivo para às pessoas com deficiência, e analisar de que forma o princípio da não-discriminação pode, se tomado como referência, contribuir para a reflexão ética e política sobre os protocolos de racionamento.

Palavras-chave: Alocação de Recursos; Triagem; Pessoas com Deficiências; Igualdade; Covid-19

Abstract: The global health crisis caused by Covid-19 imposed a critical scenario of scarcity of medical resources. In these circumstances, persons with disabilities are more vulnerable than they would be under normal circumstances. In light of this reality, we propose to present some criteria for the allocation of resources used during the crisis, highlighting their harmful nature for people with disabilities, and to analyze how the principle of nondiscrimination can, if taken as a reference, contribute to the ethical and political reflection on the rationing protocols.

Keywords: Resource allocation; Triage; Persons with Disabilities; Equality; Covid-19

\section{Por que critérios éticos e políticos?}

A crise sanitária deflagrada pela pandemia do vírus Covid-19 atingiu patamares críticos em diversos países ${ }^{1}$, tendo levado ao limite pessoal e material diferentes sistemas de saúde mundo afora ${ }^{2}$. Dentre os problemas desencadeados pela crise, constam a escassez de

\footnotetext{
${ }^{1}$ VITORIO, T.; ROVAROTO, I. 1 milhão de infectados e 56 mil mortos: um retrato do coronavírus nos EUA. Exame, [S.l.], 28 abr. 2020. Mundo. Disponível em: https://exame.abril.com.br/mundo/1-milhao-de-infectadose-56-mil-mortos-um-retrato-do-coronavirus-nos-eua/. Acesso em: 28 abr. 2020. LAUDETE, C.; PINEDO, E. Espanha tem aumento de mortes; total supera 19 mil. Terra, 16 abr. 2020. Coronavírus. Disponível em: https://www.terra.com.br/noticias/coronavirus/espanha-tem-aumento-de-mortes-total-supera-19mil,03a0925488465e166af6beodfb758azc8biz6jrc.html. Acesso em: 28 abr. 2020.

${ }^{2}$ AMARO, S. Lacking beds, masks and doctors, Europe's health services struggle to cope with the coronavirus. CNBC, [S.l.], o3 apr. 2020. Health and Science. Disponível em: https://www.cnbc.com/2020/04/03/coronavirusitaly-spain-uk-health-services-struggle-to-cope.html. Acesso em: 28 abr. 2020. GALLÓN, N. Bodies are being left in the streets in an overwhelmed Ecuadorian city. CNN, [S.l.], 04 apr. 2020. Disponível em:
}

\footnotetext{
*Doutoranda em Direito na Universidade Federal do Rio de Janeiro, Rio de Janeiro, RJ. E-mail: luana.adriano88@gmail.com ORCID: https://orcid.org/oooo-0002-6761-837X

** Mestrando em Direito na Universidade Federal do Rio de Janeiro, Rio de Janeiro, RJ. E-mail: arthurcezar@id.uff.br ORCID: https://orcid.org/oooo-0002-7747-9764

${ }^{* * *}$ Professora Adjunta de Direito da Universidade Federal do Rio de Janeiro, Rio de Janeiro, RJ. E-mail: anapbarbosa@direito.ufrj.br ORCID: https://orcid.org/oooo-0001-6331-1023
} 
recursos e a sobrecarga dos sistemas, o que pode dar ensejo ao estabelecimento de critérios para racionamento dos recursos médicos e para o atendimento seletivo de pacientes 3 . Para os sujeitos com deficiência, as preocupações não se restringem à sua qualificação nos grupos de riscos, às complicações para a realização do isolamento no caso de algumas condições e às dificuldades associadas à situação de sujeitos institucionalizados. Envolvem também a formatação dos protocolos de emergência diante da escassez de recursos - sobretudo de respiradores e leitos em UTIs (Unidades de Terapia Intensiva) de alta complexidade - que, de forma implícita e explícita, desfavorece pessoas com deficiência. A questão que anima esse tipo de triagem pode ser assim posta: "Quando o número de pessoas que precisam de determinados recursos de saúde é maior do que o número de recursos existentes, como decidir quem os recebe e quem não os recebe?".

Para responder a esse questionamento, têm sido estruturadas listas de prioridades, que categorizam, a partir de determinadas condições, as preferências no recebimento de recursos. Apesar de apresentarem critérios positivos - ou seja, o que fazer quando um paciente é prioritário -, esses protocolos também apresentam critérios negativos, de exclusão - ou seja, o que deixar de fazer quando um paciente não é preferencial. Nessas circunstâncias, pessoas com deficiência são, quando não explicitamente, implicitamente excluídas da alocação dos recursos, gerando situações de discriminação e injustiça.

A questão do racionamento justo durante uma pandemia é complexa e repleta de preocupações éticas. Ao mesmo tempo, pensar esses protocolos eticamente também demanda pensá-los politicamente. É dizer: além de ser necessário analisar critérios mais eticamente sensíveis à questão da deficiência, essa análise estaria incompleta se não buscasse entender como a criação de protocolos excludentes é possível em uma sociedade já estruturada. Dessa maneira, o desafio ético é concomitante a um desafio político: quais são as estruturas políticas que permitem gerar protocolos excludentes? Assim, nossa análise tem duas faces complementares. Primeiramente, pensamos sobre o impacto, para pessoas com deficiência, causado por determinados critérios éticos de estruturação de protocolos de distribuição de recursos. Em segundo lugar, pensamos como a formatação política da deficiência nos acordos sociais caracteriza as próprias condições de possibilidade do surgimento desses critérios - pela ausência de representação, pela desconsideração de situações de escassez aguda e pela consideração da deficiência como uma desigualdade natural, desprovida da dinamicidade do modelo social de deficiência.

\section{Critérios éticos de racionamento}

Embora a discussão sobre a natureza antiética de alguns protocolos ainda não tenha se instaurado em alguns países, em outros, vê-se a institucionalização de critérios que discriminam diretamente pessoas com deficiência, por motivos que carecem de razoabilidade depois de breve análise. Nesse sentido, nos EUA, citemos os planos estruturados pelo Estado de Alabama, anunciado em março de 2020, no qual indivíduos com deficiências mentais graves ou profundas foram considerados "candidatos improváveis ao suporte ventilatório"4. Do mesmo modo, as diretrizes estaduais de Washington

https://edition.cnn.com/2020/04/o3/americas/guayaquil-ecuador-overwhelmed-coronavirus-intl/index.html. Acesso em: 28 abr. 2020.

3 As recomendações clínicas de natureza ética, emitidas pela SIAARTI para orientar a alocação de cuidados de tratamento intensivo, ilustram esse fenômeno. cf. VERGANO, M. et. al. Clinical ethics recommendations for the allocation of intensive treaments, in exceptional, resource-limited circumstances. [S.l.]: SIAARTI, 16 mar. 2020.

4 PATHAK, Parag. et al. Leaving No Ethical Value Behind:Triage Protocol Design for Pandemic Rationing, 4. 
recomendaram que pacientes hospitalares com "perda de reservas de energia, capacidade física, cognição e saúde geral" fossem colocados em cuidado ambulatorial ou em sistema de cuidados paliativos. Ocorre que colocar certos grupos em condições naturalmente inferiores às dos demais, desconsiderando-se a desigualdade de suas situações, pode constituir uma violação do princípio da igualdade e da não-discriminação.

À luz dessa realidade, apresentaremos, na presente seção, os critérios de racionamento aparentemente neutros que foram analisados por Deborah Hellman e Kate M. Nicholson 5 ; tais critérios, como elas afirmam, se encontram presentes em protocolos estatais de racionamento e, de forma previsível, produzirão discriminações com base em deficiências ${ }^{6}$.

\section{Utilitarismo}

Os protocolos analisados por Hellman e Nicholson foram elaborados com base em princípios utilitaristas e destinavam-se a regular a distribuição de ventiladores em circunstâncias de racionamento ${ }^{7}$. Quando de sua análise, as autoras deram ênfase às orientações desenvolvidas na cidade de Nova Iorque, uma vez que, por um lado, era a cidade com o maior número de casos da doença e seu sistema de saúde se encontrava sobrecarregado; por outro, o documento em questão, isto é, as Diretrizes dos Ventiladores de Nova Iorque (New York Ventilators Guidelines), havia sido adotado há pouco ${ }^{8}$.

O ideal que permeia tais protocolos é sintetizado pelas autoras nos seguintes termos: "Seu objetivo explicitamente estabelecido é salvar tantas vidas quantas forem possíveis"9. Dessa forma, trata-se de lidar com meios limitados, diante da crise e da escassez, para que tal resultado seja atingido. Para ilustrar a aplicação desse princípio, as autoras ${ }^{10}$ fazem menção a um documento produzido pela Universidade de Washington para lidar com o racionamento; trata-se de um documento cujo fundamento é o referencial utilitarista, expresso no próprio texto, como a busca, mediante a tomada de decisões, do maior bem em favor da maior quantidade de pessoas ${ }^{11}$.

Problemas de ordem ética e jurídica são identificados por Hellman e Nicholson ${ }^{12}$ na aplicação desses protocolos: por um lado, põem-se sob risco direitos e garantias de pessoas com deficiência; por outro, desvia-se a finalidade corriqueira da prática médica, que se centra no cuidado de vidas particulares ${ }^{13}$.

A seguir, tratamos dos princípios que norteiam tais protocolos, examinados criticamente por Hellman e Nicholson, reúnem quatro aspectos: Eficiência; Probabilidade de benefícios; Expectativa de vida; Padronização e qualidade de vida.

\section{Eficiência}

A eficiência é um dos critérios identificados por Nicholson e Hellman. Refere-se à alocação dos ventiladores e sua finalidade é salvar o maior número de pessoas possível ${ }^{14}$.

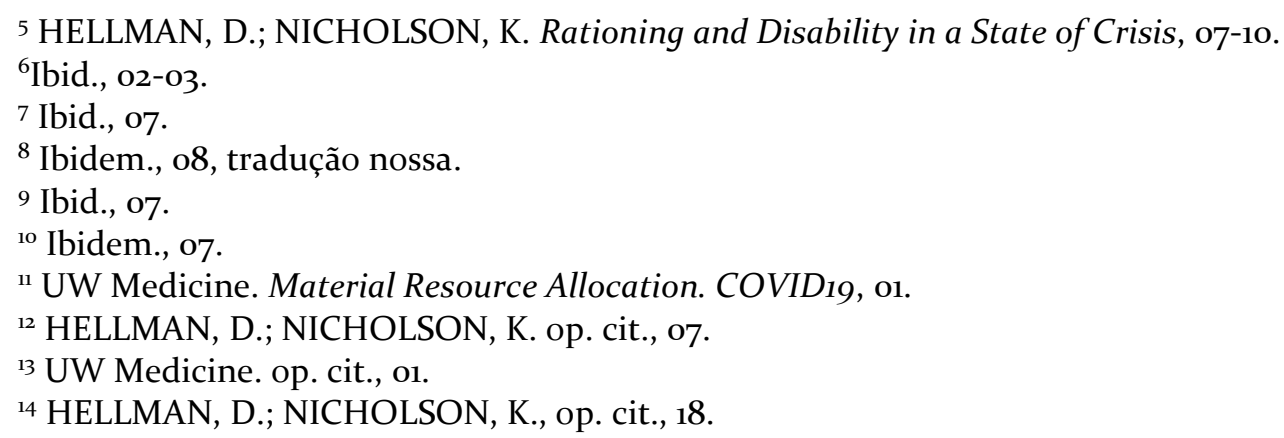


Nesse sentido, mencione-se o procedimento de periódica verificação dos pacientes, que visa a apurar os efeitos do ventilador sobre sua condição enferma; isso se dá de modo que, caso não haja melhora ou se verifique o agravamento do quadro no momento de check-in, o ventilador possa ser realocado para um novo paciente ${ }^{15}$.

Nicholson e Hellman assinalam que a realocação se dá, por determinação do protocolo de Nova Iorque, com base no princípio de racionamento da eficiência, uma vez que ele se utiliza da parametrização do tempo em que o paciente deve obter melhora ao fazer uso do ventilador, sob o risco de que, caso não melhore, ocorra a realocação. Tendo em vista que o uso do ventilador pode ser necessário para certas pessoas por mais tempo para que atinjam a mesma melhora que outros, tal critério é qualificado como moralmente questionável por Nicholson e Hellmann. Sob tais circunstâncias, os pacientes que dependem dos ventiladores por menos tempo são beneficiados ${ }^{16}$. Além disso, esse tipo de critério trata de maneira igual a alocação e a realocação de um recurso como os respiradores. É dizer: não haveria diferença moral entre reter um recurso demandando porque o demandante dele precisará por um período elevado de tempo e retirar um recurso porque o paciente que o utiliza já o faz por um tempo considerado excessivo.

A tarefa que se impõe, segundo entendem Nicholson e Hellman, é traçar quais são os limites legais aos efeitos discrepantes causados pela aplicação ${ }^{17}$. Se, por um lado, a preservação do maior número de vidas é defensável, por outro, a aplicação do princípio causaria expressivo prejuízo às pessoas com deficiência, haja vista lhes vedar o acesso a um tratamento de que necessitam - trata-se de um "close case"18. Dessa forma, Nicholson e Hellman ${ }^{19}$ sugerem, embora de forma provisória, que o princípio da equidade - presente em documentos como a Lei Brasileira de Inclusão (LBI) e a Convenção de Direitos das Pessoas com Deficiência (CDPD) - impede a aplicação do critério da eficiência.

\section{Probabilidade de benefício}

A probabilidade do benefício é um critério que confere prioridade às pessoas que possam tirar deles o maior proveito. Isto é, os grupos que têm maior prioridade são os que efetivamente têm necessidade de usar os aparelhos e, igualmente, têm maiores chances de se curar mediante o uso ${ }^{20}$. Dentro desse parâmetro, "a triagem requer a identificação de fatos clinicamente relevantes sobre pacientes individuais e sua probabilidade de se beneficiar dos recursos disponíveis" ${ }^{21}$. Dessa maneira, tem-se a impressão de maior correção, dado que a menor idade e a ausência de deficiência não são consideradas automaticamente como prioritárias em relação a outros casos, visto que o que é realmente relevante é a possibilidade de benefíción ${ }^{22}$.

A avaliação da prioridade que cabe a cada um dos pacientes segue, na cidade de Nova Iorque, o escore SOFA ("Sequential Organ Failure Assessment") ${ }^{23}$, o qual contabiliza uma pontuação que expressa as medidas clínicas de função do cérebro, da pressão sanguínea, da

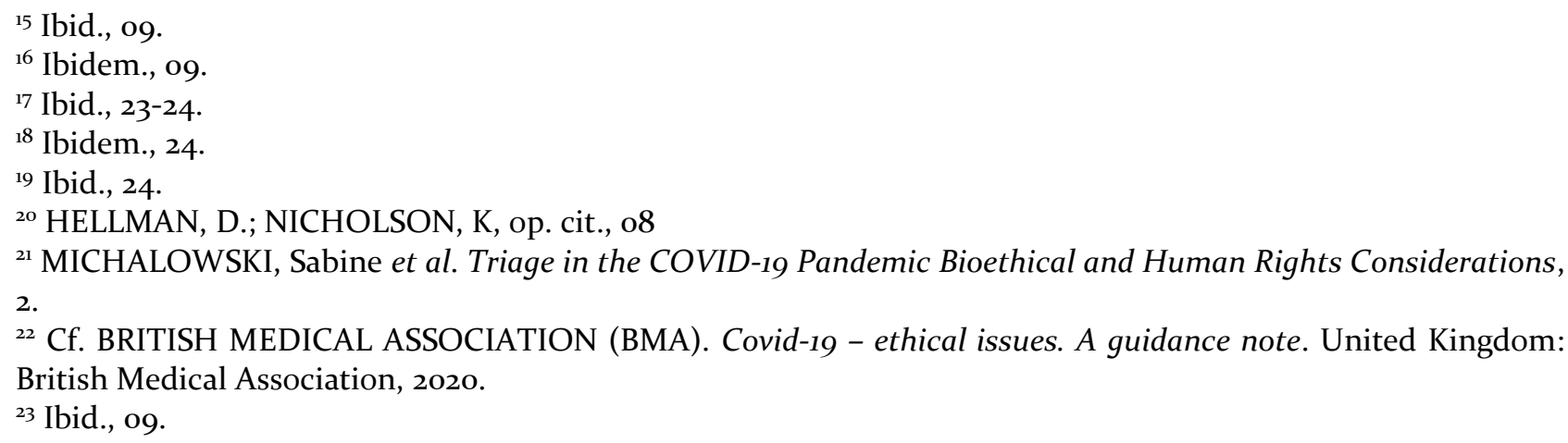


coagulação sanguínea, dos pulmões, dos rins e do fígado ${ }^{24}$. Embora não haja expressa discriminação no critério, Hellman e Nicholson ${ }^{25}$ compreendem que pessoas com deficiência podem ser por ele prejudicadas quando, em virtude da sua deficiência, tiverem menores chances de sobrevivência, ainda que utilizem ventiladores, do que pessoas que não possuem qualquer deficiência.

Às mesmas conclusões que Nicholson e Hellman chegaram ao ponderarem sobre o princípio da eficiência são aplicáveis ao princípio da probabilidade do sucesso. Contudo, há uma diferença relevante entre o princípio da eficiência e o da probabilidade do sucesso. Enquanto o primeiro desses comanda a justificativa de retenção (fundamentando, nesse sentido, a proposição: "lhe entregaremos este ventilador porque, no segundo se o fizermos, nós poderemos salvar somente uma pessoa; mas se o entregarmos para outro, poderemos salvar quatro pessoas"), o segundo promove combinações quantitativas dois a dois, mesmo que um conjunto maior seja o considerado (fundamentando a proposição "nós não lhe entregaremos este ventilador porque você só tem 30\% de chances de sobreviver; mas, se o entregarmos a outra pessoa, ela terá $80 \% ")^{26}$.

Se, por um lado, a aplicação do primeiro implica em que quatro pessoas sejam salvas ao invés de uma, a aplicação do segundo princípio promove a circunstância em que uma pessoa seria escolhida em detrimento da outra; na primeira hipótese, a cada uma das vidas é atribuído o mesmo valor, enquanto, na segunda, se consumada a entrega a quem tem maior probabilidade de benefício, essa igualdade não se verifica ${ }^{27}$. Assim, não obstante a aplicação do princípio da probabilidade de sucesso possa salvar mais vidas, o custo causado pelo acesso desigual ao tratamento médico torna o segundo princípio ainda mais problemático em termos morais do que o primeiro ${ }^{28}$.

Por último, os conhecimentos que fundamentariam a aplicação do princípio da probabilidade de sucesso (os fatos clinicamente relevantes) são passíveis de questionamento ${ }^{29}$, vez que, no contexto da pandemia, as avaliações necessárias para a tomada de decisão são mais duvidosas e provisórias do que habitualmente. Considerando esse caráter provisório, é possível supor que "o grau de confiança que os tomadores de decisão estão justificados a atribuir a dados sobre a probabilidade de sobrevivência da Covid-19, referentes a diversas categorias de pessoas, é especialmente baixo"30.

\section{Expectativa de Vida}

O critério da expectativa de vida implica o remanejamento de recursos para aqueles que, após o tratamento, teriam mais chances de viver mais ${ }^{31}$. Tal critério, por um lado, de forma geral, beneficia as pessoas mais jovens; por outro, dá margem para que sejam priorizadas as pessoas sem deficiência em detrimento de pessoas cuja deficiência torna sua expectativa de vida menor do que a média. Isso não ocorre apenas por fatores orgânicos, afetos à deficiência, mas também por fatores estruturais, de negação às pessoas com deficiência de importantes políticas sociais.

\footnotetext{
${ }^{24}$ New York State Task Force on Life and the Law; New York State Department of Health. Ventilator Allocation Guidelines, 14 .

${ }^{25}$ HELLMAN, D.; NICHOLSON, K., op. cit., 18.

26 Ibidem., 24.

27 Ibid., 24.

28 Ibidem., 24.

29 Ibid., 24.

$3^{0}$ Ibidem, 24-25, tradução nossa.

${ }^{31}$ Ibid., 18.
} 
Se eventual discriminação por razões de idade propõe-se a priorizar os mais jovens porque eles ainda não desfrutaram da vida, enquanto presume-se que os mais velhos já o fizeram, Hellman e Nicholson ${ }^{32}$ compreendem que tais justificativas não poderiam ser utilizadas em detrimento de pessoas com deficiência não idosas. Contudo, um princípio de alocação que tenha por referência tão somente a expectativa de vida ignora as características peculiares dos pacientes, uma vez que almeja simplesmente a maximização de anos salvos. Nesse quadro, compreendem as referidas autoras ${ }^{33}$ que também deve ser levado em conta o modo como os anos de vida podem ser distribuídos, considerando que tentar conferir dois anos de vida a mais para um jovem com baixa expectativa de vida pode ser mais desejável, à luz do prisma moral, do que conferir 10 anos a mais para um idoso ${ }^{34}$.

Hellman e Nicholson consideram que, caso o critério priorize as pessoas que têm maior expectativa de vida, há grandes chances de que as pessoas com deficiência sejam impactadas de forma discrepante. Entende-se que o critério de expectativa de vida é insuficiente para justificar tal impacto, caso não sejam consideradas maiores informações sobre a forma de distribuição dos anos de vida35. Assim, embora a abordagem possa parecer atraente à primeira vista, é necessário considerar também a incerteza e a subjetividade dos prognósticos, os quais podem vir a ser baseados em deficiências subjacentes, o que levantaria questões de discriminação ${ }^{36}$.

\section{Padronização e qualidade de vida}

Por esse critério, a alocação de recursos deve ser feita para pessoas cujos anos de vida sejam mais ajustados à qualidade após o tratamento ${ }^{37}$. O argumento da qualidade de vida, para Hellman e Nicholson, representa o critério mais fácil de se criticar do ponto de vista ético ${ }^{38}$. Isso porque todo tipo de avaliação da qualidade de vida feita de forma alienada à própria experiência de deficiência será pautada pelos "mitos, medos e estereótipos" que as próprias leis antidiscriminatórias se destinam a corrigir. Além disso, as medidas de qualidade são moralmente problemáticas porque expressam inadequadamente mensagens negativas sobre o valor das vidas com deficiência.

Mais importante ainda, aplicá-las ao racionamento provavelmente terá o efeito de negar às pessoas com deficiência uma oportunidade justa de se beneficiar do tratamento que salva vidas. As autoras argumentam que as medidas pautadas na qualidade de vida desfavorecem às pessoas com deficiência na medida em que uma vida com deficiência é, no geral, considerada de qualidade inferior à de uma pessoa sem deficiência39. Isso se dá especialmente pela consideração de que a deficiência é uma tragédia pessoal, que naturalmente marca vidas ruins. A contestação de que a deficiência conduza a vidas naturalmente prejudicadas é feita especialmente em Barnes ${ }^{40}$, que parte da evidência testimonial dos relatos de pessoas com deficiência, cuja autoavaliação sobre a própria qualidade de vida não é negativa.

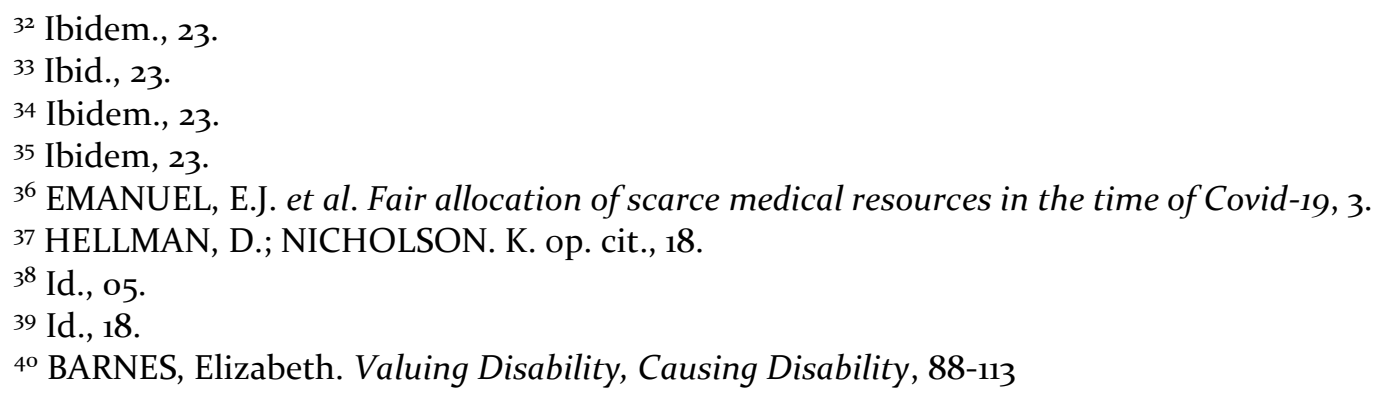


A relatividade dessas avaliações e seu potencial desfavorecimento em relação à deficiência é patente mesmo quando se tenta fornecer parâmetros aparentemente neutros e empíricos de análise. Isso porque, quando respondemos ao que parecem ser perguntas puramente empíricas, deveremos considerar: primeiro, o poder dos estereótipos sobre a percepção médica acerca dos pacientes com deficiência ${ }^{41}$; segundo, a limitação quanto ao tempo e informações durante uma emergência ${ }^{42}$. No caso do segundo fator, ressaltamos a ausência de conhecimento das consequências da doença a longo prazo.

Hellman e Nicholson argumentam ainda que, mesmo que (ou quando) a vida com deficiência seja qualitativamente pior, de modo que a escolha seja favorecer uma pessoa com maior qualidade de vida em detrimento de outra, essa política parece acumular ou aumentar a desvantagem de uma pessoa já desfavorecida. Na medida em que existem diferenças na qualidade de vida entre pessoas com e sem deficiência, fatores sociais como a discriminação parecem desempenhar um papel significativo. Assim, considerando que o objetivo das proscrições de discriminação seja o favorecimento das condições de igualdade real, usar o critério da qualidade de vida conduziria ao oposto dessa meta; ou seja, ao aumento das disparidades e vulnerabilidades 43 .

\section{Discriminação contra pessoas com deficiência}

Com o brotar da pandemia, Estados e entidades individuais passaram a instituir políticas de escassez que discriminam direta ou indiretamente pessoas com deficiência. $\mathrm{O}$ efeito discriminatório se dá, sobretudo, com a conexão entre as disposições de nãofornecimento ou retirada de recursos e a condição orgânica de deficiência. Em atenção a essa situação, instituições especializadas na ética médica têm enfatizado que as decisões tomadas nesse contexto precisam dar "devida consideração à necessidade de eliminar a discriminação ilegal, promover a igualdade de oportunidades e reduzir as desigualdades na saúde" 44 . Assim, ao elaborar critérios de triagem e cuidados específicos, é necessário evitar práticas que discriminem ilegal ou antieticamente as pessoas em grupos protegidos, como pessoas com deficiência 45 . Tal proibição de discriminação se torna mais controversa ao pensarmos que a alocação de recursos considera, no geral, exclusivamente critérios clínicos, que poderão fornecer análises a priori. Como alertado por Chisholm, uma "abordagem baseada unicamente em fatores clinicamente relevantes pode, estatisticamente, priorizar os mais jovens e, quando clinicamente relevante, pode discriminar aqueles com condições de saúde subjacentes" 46 .

É preciso considerar esses fatores sobretudo ao apontarmos parâmetros como a deficiência e a idade para estruturar a ordem de prioridades. Essas condições de vida podem já estar associadas indiretamente a outros parâmetros que avaliem a saúde de um indivíduo,

\footnotetext{
${ }^{41}$ BAGENSTOS, Samuel., May Hospitals Withhold Ventilators from COVID-19 Patients with Pre-Existing Disabilities? Notes on the Law and Ethics of Disability-Based Medical Rationing, 7-8.

${ }^{42}$ EMANUEL, E.J. et al. Fair allocation of scarce medical resources in the time of Covid-19, 4.

${ }^{43}$ HELLMAN, D.; NICHOLSON, K, op. cit., 22.

44 NATIONAL INSTITUTE FOR HEALTH AND CARE EXCELLENCE (NICE). COVID-19 rapid guideline: critical care, 2.

45 MICHALOWSKI, Sabine et al. Triage in the COVID-19 Pandemic Bioethical and Human Rights Considerations. Technical Report, 2.

${ }^{46}$ CHISHOLM, John. Doctors will have to choose who gets life-saving treatment. Here's how we'll do it. The Guardian, London, 1 apr. 2020. Opinion. Disponível em: https://www.theguardian.com/commentisfree/2020/apr/o1/doctors-choose-life-saving-treatment-ethical-rules. Acesso em: 25 abr. 2020.
} 
dado a possibilidade aumentada de doenças crônicas em segmentos de idosos e pessoas com deficiência. Nesse sentido, há em determinados protocolos uma discriminação indireta quando atribuem um "peso duplo" a condições de deficiência - tanto pelo diagnóstico quanto pelas condições frequentemente a ela associadas. Por outro lado, há também a possibilidade de discriminação direta, quando há exclusões categóricas baseadas em condições específicas, como "comprometimento cognitivo grave". Além da vagueza da definição do que significa ter um "comprometimento cognitivo grave", essas exclusões não se baseiam, no geral, na utilidade, na eficácia, na probabilidade de benefícios, ou na expectativa de vida, refletindo, no geral, uma avaliação qualitativa das vidas que merecem ser salvas 47 .

Por outro lado, há argumentos de que a não consideração da deficiência também pode ser responsável por gerar cenários de discriminação injusta. De acordo com Persad, isso significa que buscar salvar mais vidas, no contexto da pandemia, não significa dizer que as pessoas com deficiência sejam menos merecedoras de serem salvas. Dessa maneira, utilizar critérios baseados em efetividade ou expectativa de vida poderia ser nãodiscriminatório, desde que esses critérios sejam baseados em evidências, e não em estereótipos ${ }^{48}$. Buscar salvar mais vidas, nesse sentido, seria um princípio pautado na ideia de "each counts for one" - em alinhamento com a ideia de Parfit de que salvamos o maior número "porque damos igual peso para salvar cada um" 49.

Em Persad, o argumento de salvar mais vidas teria a consequência de salvar também mais vidas com deficiência ${ }^{50}$. Assim, no exemplo dado por Hellman e Nicholson do critério da eficiência ${ }^{51}$, uma entre quatro vidas salvas no cenário em que se aplica esse parâmetro será uma vida com deficiência, visto que, de acordo com o Censo Brasileiro de 2010, 23.9\% da população brasileira - quase um quarto - possui algum tipo de deficiência. Essa percepção pode ser contestada por injustificadamente agregar vidas de pessoas com e sem deficiência. Tendo em vista que o aumento de probabilidade de "salvação" buscado é do total de vidas salvas coletivamente, não se pode inferir da agregação que, de fato, se dá a cada pessoa, individualmente, a chance de ser salva. Assim, indivíduos com deficiência que reclamam de protocolos discriminatórios não estão demandando privilégios injustificados sobre os demais. Eles estão, em verdade, solicitando protocolos baseados em evidências que usem a avaliação individual de prospecções de sobrevivência similar à de outros sem deficiência que estão usando os ventiladores.

Apesar dos argumentos de Persad buscarem salvar mais vidas, em geral - o que inclui salvar também mais vidas de pessoas com deficiência -, é preciso considerar de que maneira as pessoas com deficiência podem participar do processo de legitimação das regras de distribuição de recursos. Isso porque qualquer perspectiva que não resguarde seus pontos de vista poderá ser discriminatória. Há dois sentidos interconectados em que essas discriminações, realizadas no contexto da pandemia, denunciam uma estrutura política excludente em relação às pessoas com deficiência - anterior inclusive ao evento pandêmico. O primeiro deles diz respeito à desconsideração da adoção do modelo social pela (Convenção de Direitos das Pessoas com Deficiência) CDPD. De acordo com Asís Roig, para

\footnotetext{
47 WOLF, Leslie; WENDY, Hensel. Valuing lives: Allocating scarce medical resources during a public health emergency and the Americans with Disabilities Act (perspective), 03.

$4^{8}$ PERSAD, Govind. Why Disability Law Permits Evidence-Based Triage in a Pandemic, 2.

49 PARFIT, Derek. Innumerate Ethics, 301, tradução nossa.

$5^{\circ}$ PERSAD, Govind. op. cit., 2.

${ }^{51}$ HELLMAN, D.; NICHOLSON, K. op. cit., 24.
} 
o modelo social, a deficiência é uma situação na qual se encontram ou podem se encontrar uma ou mais pessoas, inexistindo um traço individual que a caracterize. Além disso, a deficiência tem uma origem social, devendo a sociedade ser a destinatária das medidas voltadas para o enfrentamento das desigualdades. Dessa maneira, uma política normativa que se dirige a pessoas com deficiência em tempos de pandemia deve pautar-se na igualdade e na não-discriminação ${ }^{52}$. Um segundo sentido de denúncia dos protocolos de triagem que discriminam pessoas com deficiência trata do preenchimento do conteúdo normativo da igualdade. Pretendemos analisar de que forma a consideração política da igualdade, segundo o referencial de Rawls, pode determinar o modo como se responde a questões de escassez de recursos em pandemias. Nesse ponto, pretendemos pensar como as ideias de igualdade e discriminação da teoria de justiça subjacente à maximização dos recursos na distribuição (em Rawls, maxi-min) podem ser enviesadas quando falamos de a) pessoas não devidamente representadas na posição original; b) pessoas consideradas como naturalmente prejudicadas por sua deficiência; e c) condições de escassez moderada da circunstância objetiva de justiça, que não se compatibiliza com a escassez aguda provocada pela pandemia.

\section{Obrigação de não-discriminação e o igualitarismo em Rawls}

Asís Roig entende que a igualdade pode ser compreendida como valor, princípio ou como direito. Como diferenciação negativa, a igualdade determina quando igual tratamento deve ser dispensado, ainda que as circunstâncias ou situações sejam diferentes, porquanto tal diferença seja considerada irrelevante para o gozo ou exercício de certos direitos. Essa dimensão corresponde à não-discriminação. Quando consideradas relevantes, as diferenças de situação ou circunstância ensejam o reconhecimento da discriminação positiva, advindo dessa a função promocional ou redistributiva do reconhecimento da igualdade no âmbito do direito ${ }^{53}$. Nesse sentido, face às diferenças significativas que impedem tratar igualmente desiguais, os instrumentos jurídicos devem comportar as demandas das discrepâncias de vulnerabilidade. Em tempos de pandemia, as referidas discrepâncias podem ser atestadas pelas diferenças nos modos como pessoas com e sem deficiência são tratadas ao demandarem cuidados médicos.

Outra concepção de igualdade relevante nesse cenário é a que considera as oportunidades. Bobbio entende que o "princípio da igualdade das oportunidades, quando elevado a princípio geral, tem como objetivo colocar todos os membros daquela determinada sociedade na condição de participar da competição pela vida, ou pela conquista do que é vitalmente mais significativo, a partir de posições iguais"54. A igualdade de oportunidades opera um sentido particular na noção de "sobrevivencialismo", que modula a ideia subjacente às triagens no sentido de que "recursos devem ser destinados aos que têm mais chance de sobreviver". Bobbio, ao falar na igualdade de oportunidades como importante para criar aptidões na "competição pela vida" transparece o sentido em que se torna razoável negar oportunidades aos que têm vidas que não valem a pena ser vividas.

A igualdade entra como um princípio de estruturação da teoria de Rawls já na ideia da posição original, na qual encontram pessoas livres, iguais e racionais, preocupadas em promover seus próprios interesses, com vistas a definir os termos fundamentais de sua

\footnotetext{
${ }^{52}$ ASÍS ROIG, Rafael de. Sobre el modelo social de discapacidad: críticas y éxito, 2.

53 ASÍS ROIG, Rafael de. Sobre la discriminación positiva: especial referencia al Derecho europeo, 27-51. ASÍS ROIG, Rafael de. La Igualdad en el discurso de los derechos, 149 -168.

54 BOBBIO, Norberto. Igualdade e Liberdade, p. 30-31.
} 
associação ${ }^{55}$. A partir dessa associação em uma situação de igualdade original, modulada pelo véu da ignorância, seria possível derivar dois princípios de justiça: o princípio da liberdade e o princípio da igualdade (esse associado ainda ao princípio da diferença ${ }^{56}$ ). Portanto, há dois fatores de consideração da noção de igualdade em Rawls ao tratarmos da deficiência: o estado da posição inicial, pautado na igualdade das partes representativas da teoria de justiça, e o segundo princípio de justiça, baseado nos princípios da igualdade equitativa de oportunidades e no princípio da diferença.

\section{Igualdade na posição original e deficiência}

Na situação da posição original, há duas circunstâncias que podemos considerar como sensíveis ao momento da pandemia para as pessoas com deficiência. Primeiramente, a homogeneidade da situação entre as partes demanda que elas estejam em um "leque normal de variação" 57 . A equivalência dentro desse confere aspectos de legitimidade, reciprocidade e cooperação aos termos ajustados; contudo, sua definição não inclui pessoas com deficiência, o que é explicitado em Liberalismo Político. Como Rawls afirma: "o problema de tratamentos médicos especiais e de como cuidar dos deficientes mentais é posto de lado. Se pudermos elaborar uma teoria viável para o leque normal, podemos tentar, depois, resolver esses outros casos" 58 . Por esse motivo, Cuenca argumenta que, mesmo debaixo do véu da ignorância, os representantes podem ter assegurada sua condição de não-deficiência, pelo que a teoria rawlsiana exclui as pessoas com deficiência do debate moral59.

Protocolos que usam, como critérios de exclusão de prioridade, estruturas como a "Rockwood Frailty Scale" podem denunciar a importância de se considerar pessoas com deficiência desde a concepção das regras de preferência. Esse tipo de escala resume o nível geral de aptidão ou fragilidade de uma pessoa, variando de 1 ("Muito Ativo") a 9 ("Doente Terminal"). As pontuações são baseadas em uma avaliação de cognição e humor, mobilidade, função, saúde social, comorbidades, medicamentos e atitudes em saúde ${ }^{60}$. Por considerar significativamente a necessidade de apoio às atividades da vida diária, a escala Rockwood é altamente sensível à deficiência. Assim, o maior risco é que se exclua da preferência de tratamento uma pessoa com determinada deficiência por não conseguir gerenciar seus próprios cuidados sem apoio. Isso pode significar uma discriminação baseada na deficiência, reforçando-se a ideia de que são vidas que "valem menos". Nesse tipo de protocolo, a ausência de representatividade é sentida quando da aplicação prática de regras teoricamente igualitárias, desconsidera-se a institucionalidade pública das situações de dependência ${ }^{61}$. Assim é que, por não levarem em conta as perspectivas de pessoas com deficiência e de dependência, esses normativos não considerarão suas demandas de ajustes razoáveis, cujo suprimento (seja por meio da utilização por mais tempo, seja por alocação prioritária) é necessário para um acesso significativo à saúde. Contra esse argumento, há a noção de que os ajustes razoáveis podem esbarrar em limites de idoneidade, necessidade, proporcionalidade e aceitação, além de não poderem gerar custos desproporcionais em sua

\footnotetext{
55 RAWLS, John, Uma Teoria de Justiça, 12.

${ }^{6}$ Ibidem., 88.

57 RAWLS, John. Liberalismo Político, 68.

${ }^{8}$ Ibidem.., 325

59 CUENCA, Patrícia. Sobre la inclusión de la discapacidad en la teoría de los derechos humanos, 107-108.

${ }^{60}$ ROCKWOOD, K. et al. A global clinical measure of fitness and frailty in elderly people, 489-495.

${ }^{61}$ KITTAY, Eva Feder, Love’s Labor, 75-76.
} 
aplicação ${ }^{62}$. Esses limites, segundo uma concepção de que derivam de uma racionalidade ideal, seriam os mesmos, com ou sem a participação de pessoas com deficiência.

Um segundo fator da posição original que nos interessa, no âmbito da pandemia, diz respeito às circunstâncias de justiça que permitem a cooperação desejável na posição original. Para Rawls, existem fatores que tornam a cooperação humana simultaneamente possível e necessária, dentre elas, a circunstância da escassez moderada implícita para atender a uma ampla gama de situações ${ }^{63}$. Assim é que os recursos existentes no momento da posição original "não são abundantes ao ponto de não emergirem reivindicações conflitantes sobre a parcela que cabe a cada um de seus membros, e nem tão exíguos ao ponto de qualquer forma de cooperação ser impossível"64. Em que pese a idealidade da situação inicial, é preciso perguntar de que maneira regras pensadas em contextos de escassez moderada se aplicam a situações de escassez aguda. É possível, assim, que uma sociedade pensada sob o véu da ignorância, em que há escassez de recursos moderada, reflita sobre regras legítimas para um mundo pandêmico, em que a escassez aguda é um requisito estrutural da crise? Em resumo, teorias políticas pensadas "na normalidade" de escassez de recursos moderadas respondem eficazmente a realidades da "anormalidade" de escassez total e de banalização das escolhas trágicas?

Essa total ausência de tratamento dos que já estão em piores condições, devido à escassez aguda, considera a aplicação do "maximin", que, em Rawls, leva à maximização das perdas mínimas ${ }^{65}$ e ao atingimento das melhores condições possíveis aos menos favorecidos. Segundo essa regra, diante da incerteza, as alternativas devem ser hierarquizadas de acordo com os piores resultados possíveis, devendo a escolha ser do melhor entre esses. Nesse sentido, o princípio maximin, individualmente aplicado, diz que é preciso avaliar todas as políticas disponíveis em termos da pior possibilidade que possa ocorrer ao sujeito que avalia. Na posição original, visto que a sociedade é pensada para os "normais", os "habitualmente cooperativos", nem mesmo a escolha da melhor entre as piores alternativas de protocolos poderá priorizar determinados sujeitos com deficiência. Em um contexto de desigualdade, um sistema prioritário associado à demanda excessiva de recursos disponíveis pelos grupos de maior prioridade - mesmo sem nenhuma exclusão explícita de certos tipos de indivíduos -, sempre haverá alguns pacientes que nunca seriam tratados durante uma escassez ${ }^{66}$.

\section{Aplicação do princípio da diferença e não-discriminação}

O segundo aspecto de igualdade em Rawls que nos interessa, quando falamos de deficiência e pandemia, é na aplicação de seu segundo princípio de justiça, que engloba o princípio da igualdade equitativa de oportunidades e o princípio da diferença ${ }^{67}$ e alberga a noção de reparação das desigualdades imerecidas. Rawls menciona especificamente as desigualdades "de nascimento e de dotes naturais", as quais por não serem merecidas, devem ser compensadas. Aponta, assim, que desigualdades inatas devem ser aplainadas. $\mathrm{O}$ trato de uma pessoa com deficiência, nesse sentido, deve ser alocado no campo do "desvio das contingências em direção da igualdade". Tal perspectiva de "desigualdades naturais" é uma negação do modelo social, uma vez que, nesse, as barreiras sociais constituem-se enquanto premissas inelimináveis da desigualdade advinda da deficiência.

\footnotetext{
${ }^{62}$ ASÍS ROIG, Rafael de. Lo razonable en el concepto de ajuste razonable, 100-105.

63 RAWLS, John. Uma Teoria de Justiça, 136-137.

${ }^{6}$ VITA, Álvaro de. A tarefa prática da filosofia política de John Rawls, 15.

${ }^{65}$ RAWLS, John. Uma Teoria de Justiça, 165.

${ }^{66}$ PATHAK, Parag A et al. op. cit., 5.

${ }^{67}$ RAWLS, John. Uma Teoria de Justiça, 79.
} 
Do ponto de vista da CDPD, o meio e as barreiras nele contidas constituem-se enquanto fatores essenciais no entendimento das desigualdades correlacionadas à situação de deficiência. Rawls interpreta a deficiência como um caractere não-dinâmico, configurado enquanto um dado em si a partir do nascimento ou do acidente. A investigação se torna ainda mais complexa se se considera que estas pessoas com impedimentos imerecidos, localizadas abaixo da linha das capacidades, vieram à luz em uma sociedade pensada sob os auspícios das negociações empreendidas por representantes daqueles localizados acima da linha das capacidades.

\section{Conclusão}

Ante ao analisado, deve-se destacar três desafios que a pandemia põe para a estruturação de protocolos politicamente justos, sensíveis à deficiência: a exclusão das pessoas com deficiência da composição dos termos de justiça; a escassez não moderada, mas sim aguda de recursos; e a noção individualista da desigualdade de oportunidades ensejada pela situação de deficiência em sociedade. Ultrapassar os problemas políticos e éticos apresentados nesse trabalho é essencial para garantir que as respostas à pandemia sejam não excludentes, não discriminatórias e sensíveis à realidade de pessoas com deficiência.

\section{Referências}

AMARO, S. Lacking beds, masks and doctors, Europe's health services struggle to cope with the coronavirus. CNBC, [S.l.], o3 apr. 2020. Health and Science. Disponível em: https://www.cnbc.com/2020/o4/o3/coronavirus-italy-spain-uk-health-services-struggle-tocope.html. Acesso em: 28 abr. 2020.

ASIS ROIG, Rafael de. La Igualdad en el discurso de los derechos. In: LÓPEZ GARCÍA, J. A.; ALBERTO DEL REAL, J ( Ed.). Los derechos: entre la ética, el poder y el derecho. Madrid: Dykinson, 200o, p. 149-168.

ASIS ROIG, Rafael de. Sobre la discriminación positiva: especial referencia al Derecho europeo. In: MARIÑO MENÉNDEZ F.; FERNÁNDEZ LIESA, C. La protección de las personas y grupos vulnerables en el Derecho europeo. Madrid: BOE. Boletín Oficial del Estado, 20o1, p. 27-51.

ASÍS ROIG, Rafael de. Sobre el modelo social de discapacidad: críticas y éxito. Papeles el tiempo de los derechos. [S.1], n. 1, 2013. Disponível em: https://earchivo.uc3m.es/handle/10016/19304. Acesso em: 25 abr. 2020.

ASÍS ROIG, Rafael. Lo razonable en el concepto de ajuste razonable. In: SALMÓN, E.; BREGAGLIO, R (Eds.). Nueve conceptos claves para entender la Convención sobre los derechos de las personas con discapacidad. Lima: IDEHPUCP, 2015, p. 99-118.

BAGENSTOS, Samuel R. May Hospitals Withhold Ventilators from COVID-19 Patients with Pre-Existing Disabilities? Notes on the Law and Ethics of Disability-Based Medical Rationing, Yale Law Journal Forum, v. 130 ${ }^{68}, 24$ mar. 2020. Disponível em: https://papers.ssrn.com/sol3/papers.cfm?abstract id=3559926. Acesso em: 25 abr. 2020.

\footnotetext{
${ }^{68}$ Informamos que o volume 130 do Yale Law Journal Forum ainda será publicado, embora o artigo em questão já tenha sido disponibilizado virtualmente.
} 
BARNES, Elizabeth. Valuing Disability, Causing Disability, Ethics, Chicago, v. 125, n. 1, p. 88113 oct. 2014 .

em: https://www.jstor.org/stable/10.1086/677021?seq=1\#metadata info tab contents. Acesso em: 30 abr. 2020.

BOBBIO, Norberto. Igualdade e Liberdade. Rio de Janeiro: Ediouro, 1997.

BRITISH MEDICAL ASSOCIATION (BMA). Covid-19 - ethical issues. A guidance note. United Kingdom: British Medical Association, 2020. Disponível em: https://www.bma.org.uk/media/2226/bma-covid-19-ethics-guidance.pdf . Acesso em: 25 abr. 2020.

CHISHOLM, John. Doctors will have to choose who gets life-saving treatment. Here's how we'll do it. The Guardian, London, 1 apr. 2020. Opinion. Disponível em: https://www.theguardian.com/commentisfree/2020/apr/o1/doctors-choose-life-savingtreatment-ethical-rules. Acesso em: 25 abr. 2020.

CUENCA, Patricia. Sobre la inclusión de la discapacidad en la teoría de los derechos humanos. Revista de Estudios Políticos (nueva época). Madrid, n. 158, p. 103-137, octubrediciembre 2012.

EMANUEL, E.J. et al. Fair allocation of scarce medical resources in the time of Covid-19. The New England Journal of Medicine, [S.1.], 23 apr. 2020. Disponível em: https://www.nejm.org/doi/pdf/10.1056/NEJMsb2005114?articleTools=true. Acesso em: 30 abr. 2020.

GALLÓN, N. Bodies are being left in the streets in an overwhelmed Ecuadorian city. CNN, [S.l.], o4 apr. 2020. Disponível em: https://edition.cnn.com/2020/o4/o3/americas/guayaquilecuador-overwhelmed-coronavirus-intl/index.html. Acesso em: 28 abr. 2020.

HELMANN, D.; NICHOLSON, K. Rationing and Disability in a State of Crisis. Public Law and Legal Theory Research Paper, [S.1.], n. 2020-33, p. 01-26, apr. 2020. Disponível em: https://ssrn.com/abstract=3570088. Acesso em: 29 abr. $2020[1]$.

KITTAY, Eva. Love's Labor: essays on women, equality and dependency. New York: Routledge. 1999.

LAUDETE, C.; PINEDO, E. Espanha tem aumento de mortes; total supera 19 mil. Terra, 16 abr. 2020. Coronavírus. Disponível em: https://www.terra.com.br/noticias/coronavirus/espanha-tem-aumento-de-mortes-totalsupera-19-mil,o3aog25488465e166af6beodfb758a3c8biz6jrc.html. Acesso em: 28 abr. 2020.

MICHALOWSKI, S. et al. Triage in the COVID-19 Pandemic Bioethical and Human Rights Considerations. A Joint Technical Report of the Essex Autonomy Project \& the Ethics of Powerlessnes Project - School of Law; School of Philosophy and Art History, University of Essex, Essex, 2020.

NATIONAL INSTITUTE FOR HEALTH AND CARE EXCELLENCE (NICE). COVID-19 rapid guideline: critical care. [S.l.]: NICE, 2020. Disponível em: https://www.nice.org.uk/guidance/ng159/resources/covid19-rapidguideline-critical-care-inadults-pdf-66141848681413. Acesso em: 25 abr. 2020. 
NEW YORK STATE TASK FORCE ON LIFE AND THE LAW; NEW YORK STATE DEPARTMENT OF HEALTH. Ventilator Allocation Guidelines, [S.l.]: New York State Task Force on Life and The Law; New York State Department of Health, 2015. Disponível em: https://www.health.ny.gov/regulations/task force/reports publications/docs/ventilator gui delines.pdf. Acesso em: 28 abr. 2020.

PATHAK, Parag. et al. Leaving No Ethical Value Behind:Triage Protocol Design for Pandemic Rationing, NBER Working Papers Series, Cambridge, n. 26951, p. 01-45, apr. 2020. Disponível em: https://economics.mit.edu/files/19358. Acesso em: 28 abr. 2020.

PARFIT, Derek. Innumerate Ethics. Philosophy E Public Affairs,[S.1.], v. 7, n. 4, p. 285-301, 1978. Disponível em: https://www.jstor.org/stable/2264959. Acesso em: 30 abr. 2020.

PERSAD, Govind. Why Disability Law Permits Evidence-Based Triage in a Pandemic. Yale Journal Forum, [S.1.], v. 130, U Denver Legal Studies Research Paper n. 20-10, 8 apr. $2020^{69}$. Disponível em: https://papers.ssrn.com/sol3/papers.cfm?abstract id=3571139. Acesso em: 30 abr. 2020.

RAWLS, John. Uma teoria de Justiça. Trad. Almiro Pisetta e Lenitta M. R. Esteves. São Paulo: Martins Fontes. 1997.

RAWLS, John. O liberalismo político. Trad. Dinah de Abreu Azevedo. 2. ed. 2. Imp. São Paulo : ed. Ática. 2000.

ROCKWOOD, K. et al. 2005. A global clinical measure of fitness and frailty in elderly people. Canadian Medical Association Journal. [S.1.], v. 173, Issue 5, p. 489-495, aug. 2005. Disponível em: https://www.cmaj.ca/content/cmaj/173/5/489.full-text.pdf. Acesso em: 30 abr. 2020.

UW Medicine. Material Resources Allocation and Principles. Covid-19 outbreak. [S.1.]: UW Medicine, 05 apr. 2020. Disponível em: https://covid19.uwmedicine.org/Screening\%2oand\%2oTesting\%2oAlgorithms/Other\%2oInpatient\%2oCli nical\%2oGuidance/Clinical\%20Care\%20in\%2oICU/Material\%2oResource\%2oAllocation.CO VID19.docx. Acesso em: 28 abr. 2020.

VERGANO, M. et. al. Clinical ethics recommendations for the allocation of intensive treaments, in exceptional, resource-limited circumstances. [S.l.]: SIAARTI, 16 mar. 2020. Disponível em: http://www.siaarti.it/SiteAssets/News/COVID19\%20\%20documenti\%2oSIAARTI/SIAARTI\%20-\%20Covid-19\%20-

\%20Clinical\%20Ethics\%2oReccomendations.pdf. Acesso em: 28 abr. 2020.

VITA, Álvaro de. A tarefa prática da filosofia política em John Rawls. Lua Nova: Revista de Cultura e Política, São Paulo, n. 25, p. 05-24, abr. 1992.

VITORIO, T.; ROVAROTO, I. 1 milhão de infectados e 56 mil mortos: um retrato do coronavírus nos EUA. Exame, [S.1.], 28 abr. 2020. Mundo. Disponível em: https://exame.abril.com.br/mundo/1-milhao-de-infectados-e-56-mil-mortos-um-retrato-docoronavirus-nos-eua/. Acesso em: 28 abr. 2020.

WOLF, Leslie; WENDY, Hensel. Valuing lives: Allocating scarce medical resources during a public health emergency and the Americans with Disabilities Act (perspective). PLoS Currents, [S.1.], 21 sep. 2011. Disponível em: https://www.ncbi.nlm.nih.gov/pmc/articles/PMC3199960/?report=reader. Acesso em: 30 abr. 2020.

\footnotetext{
${ }^{69}$ Esclarecemos que, embora o artigo já tenha sido disponibilizado virtualmente, o v. 130 do Yale Journal Forum
} ainda não foi publicado. 